\title{
Reliability of cutaneous landmarks in \\ catheter length assessment during PICC insertion.
}

S. Elli ${ }^{\circ}$, L. Cannizzo ${ }^{\circ}$, S. Vimercati ${ }^{\circ}$, L. Giannini ${ }^{\circ}$, A. Siligato $\$$, V. Pigozzo*, G. Bellani ${ }^{\circ}$, A. Lucchini ${ }^{\circ}$

- General Intensive Care Unit - Picc Team - ASST Monza - S.Gerardo Hospital, University of Milano-Bicocca

* Neurosurgical Intensive Care Unit - Picc Team - ASST Monza - S.Gerardo Hospital, University of Milano-Bicocca

$\$$ Infectious Diseases Unit - Picc Team - ASST-Monza - S.Gerardo Hospital, University of Milano-Bicocca

Backgrond: the "central catheter" feature is strictly dependent on the correct position of the PICC's tip, so it is important to have an estimated measure of the catheter to reach the cavo-atrial junction (CAJ). Especially for "distal trimming PICCs", the reliability of cutaneous landmarks thus becomes a determining factor for correct positioning and proper catheter management.

Aim: Evaluate the reliability of cutaneous landmarks in catheter length assessment during PICC insertion.

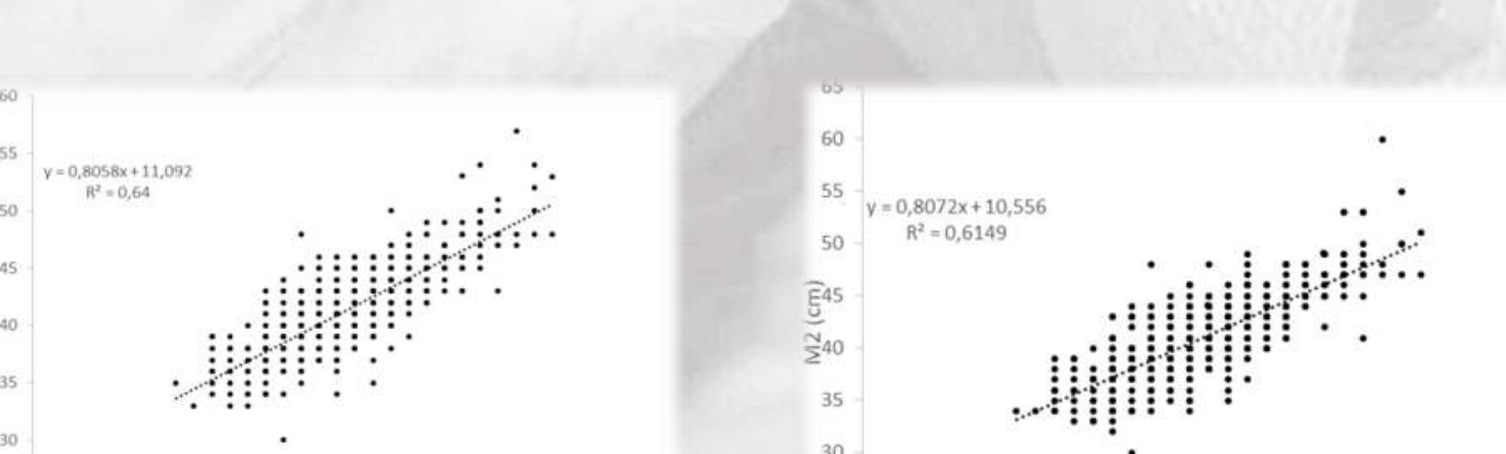

\begin{tabular}{|c|c|c|c|c|c|c|c|c|c|}
\hline & Age & Weight & Height & BMI & $\begin{array}{c}\text { Length } \\
\text { EK } \\
\text { guided }\end{array}$ & Length M1 & Length M2 & $\begin{array}{c}\text { Bias } \\
\text { EKG/M1 }\end{array}$ & $\begin{array}{c}\text { Bias } \\
\text { EKG/M2 }\end{array}$ \\
\hline MeantSD & $59,7 \pm 16,3$ & $65 \pm 7,1$ & $166,2 \pm 9,6$ & $24,9 \pm 5,4$ & $37,7 \pm 3,8$ & $43,6 \pm 7,2$ & $43,6 \pm 4,2$ & $3,77 \pm 2,44$ & $3,28 \pm 2,57$ \\
\hline Median & 61,6 & 65,0 & 165,0 & 24,4 & 38,0 & 43,0 & 45,0 & 4,00 & 3,00 \\
\hline Q1 & 49,9 & 62,5 & 159,3 & 21,3 & 35,0 & 42,0 & 42,0 & 2,00 & 2,00 \\
\hline Q3 & 71,9 & 67,5 & 172,0 & 27,7 & 40,0 & 45,0 & 46,0 & 5,00 & 5,00 \\
\hline
\end{tabular}

Results: Both $\mathrm{M} 1$ and $\mathrm{M} 2$ were significantly associated with EKG guided, but the regression line differed from identity indicating a bias between $\mathrm{M} 1 / \mathrm{M} 2$ and EKG. The average bias between $\mathrm{M} 1 / \mathrm{M} 2$ and EKG guided catheter length were $3.77 \pm 2.44 \mathrm{~cm}$ (IC 95\%: $8.56-1.02$ ) and $3.28 \pm 2.57 \mathrm{~cm}$ (IC $95 \%: 8.32-1.75)$ respectively.
Medods: in 530 PICC insertions, from 2014 December to 2016 October, Picc Team ASSTMonza recorded estimated length by cutaneous landmarks and effective lenght by EKG guided tip location. Two distinct techniques were used (we called them M1 and M2). All data were stored in Picc Team ASST-Monza database and analyzed by SPSS ver. 24. The first assessment (M1) is obtained measuring the distance from exit site to the omolateral clavicle-sternal articulation and adding $10 \mathrm{~cm}$ for the access from the right arm or $15 \mathrm{~cm}$ for the access from the left arm (Ocado Technique). The second assessment (M2) is obtained measuring the distance from exit site to the middle of clavicle and adding the distance from the middle of clavicle to the third intercostal space at the right sternal border.

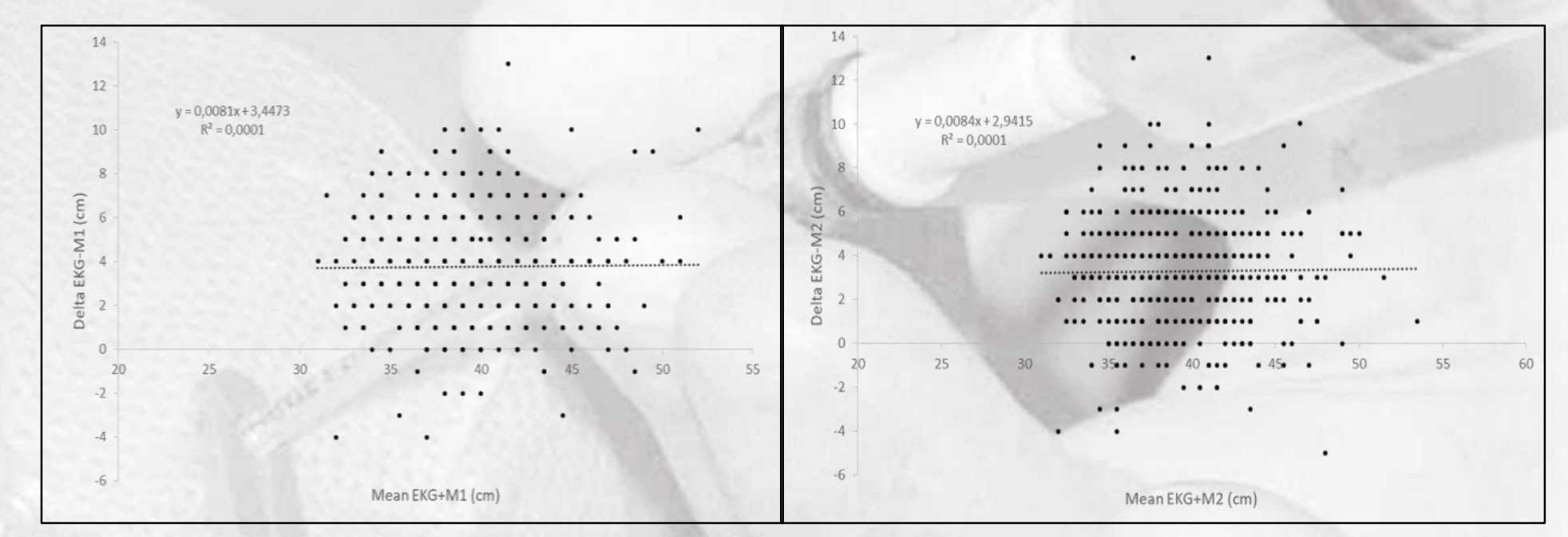

Conclusion: Cutaneous landmarks with post procedural tip location techniques, could easily determine an over-advanced positioning of the catheter, so we have two chances: tolerate a suboptimal tip location (with increased thrombotic risk and arrhythmias) or retract the catheter (with increased infective risk, additional manipulation and delayed time of insertion). Q1 of the average error on $\mathrm{M} 1$ and $\mathrm{M} 2(2 \mathrm{~cm})$ indicates that arbitrarily subtracting $3-4 \mathrm{~cm}$ from the preventive measures does not always provide a more precise indication. Cutaneous landmarks in PICC insertion appeared unreliable, if associated to post procedural tip location. They may be useful in EKG guided tip location, for increase security and optimize the "out of skin" catheter tract. 